Verfassers zu handeln. ${ }^{2}$ Dabei ist offenbar nicht einmal der Versuch unternommen worden, den Lesern einigermaßen aktuell zu informieren.

Insofern ist zu empfehlen, in den Jahresberichten von ai direkt nachzusehen. ${ }^{3}$ Hernekamp $p^{4}$ ist - nach nunmehr acht Jahren! - beizupflichten: Neues von Heinz in keiner Beziehung zu erwarten.

Niels Lau

\title{
Ludger Kühnhardt
}

Die Universalität der Menschenrechte -

Studie zur ideengeschichtlichen Bestimmung eines politischen Schlüsselbegriffes Günter Olzog Verlag, München, 408 S., DM 68,-

Ludger Kühnhardt ist Politologe und Historiker. Seit 1977 hat der »jüngste Privatdozent seit Kriegsende« in Bonn (so die Verlagswerbung) Bücher u.a. über christliche Soziallehre, Entwicklungspolitik und Flüchtlingsprobleme veröffentlicht und ist als Journalist hervorgetreten. Die vorliegende Studie stellt seine im Jahre 1986 in Bonn angenommene Habilitationsschrift dar, die von Karl Dietrich Bracher betreut worden ist.

Der Titel weckt große Erwartungen. Die Menschenrechte sind in Europa entstanden - bei allem Schillern des Begriffs läßt sich das sagen. Ungeachtet dieser Genese bestreitet heute kein Staat und kein Denker, daß Menschenrechte ein hohes Gut sind. Alle Staaten identifizieren sich mit der Allgemeinen Erklärung der Menschenrechte der Vereinten Nationen, auch wenn sie keineswegs selten nicht nach ihr handeln; viele Staaten haben sich völkerrechtlich verpflichtet, einzelne, in Verträgen meist konkreter gefaßte Menschenrechte einzuhalten. Mehrere Verfassungen in aller Welt beziehen sich auf die Allgemeine Erklärung, sehr viele enumerieren selbst einzelne Menschenrechte. Dieser Konsens verdeckt freilich wie allgemein bekannt ist - mannigfache Dissense en detail und im Grundsätzlichen: Warum Menschenrechte gelten, wer sich auf sie berufen kann, was zum Katalog "der" Menschenrechte gehört und gehören sollte - alles dies ist heillos umstritten, auch wenn der institutionelle Menschenrechtsschutz im UN-System, vor allem aber in den kontinentalen Schutzsystemen Europas und Amerikas gewisse Erfolge zeitigt und dies im kontinentalen System Afrikas vielleicht bevorsteht.

Setzt nun jemand an, die "Universalität der Menschenrechte « nachzuweisen, so kann man sich das auf unterschiedliche Art vorstellen. Er könnte von der Geschichte eines politi- 
schen Begriffs handeln oder Rechtsgeschichte betreiben, mit dem Blick auf Völkerrecht oder innerstaatliches Recht oder ihre Bezüge zueinander. Es wäre dies der Bericht über die fortschreitende rechtliche Umsetzung einer Idee auf universaler Ebene. Kühnhardt liefert dazu Elemente. Recht eigentlich geht es ihm aber um anderes: „Universalität« heißt für ihn "Geeignetheit « für die politischen Systeme aller Völker und Zeiten. Dem Befund folgt der Anspruch: Die Menschenrechte, ein ihren Vorgaben genügender Entwurf vom Staat, sollen in alle Welt getragen werden - der "Schlüsselbegriff" Menschenrechte soll alle Pforten aufschließen, hinter denen Individuen schmachten.

Daß die Staaten ihre nationalen Rechtsordnungen und ihr Handeln gegenüber Individuen an einem einheitlichen Maßstab auszurichten hätten, könnte eine juristische These sein. Dann müßte der Nachweis geführt werden, daß dem geltenden Völkerrecht derartige Maßstäbe zu entnehmen wären. Solange, wie erwähnt, über den Geltungsgrund der Menschenrechte, über die Funktionen und Inhalte einzelner Menschenrechte gestritten wird, solange ferner die Ausgestaltung der innerstaatlichen Ordnungen im Grundsatz jedem souveränen Staat überlassen bleibt und lediglich staatliche Einzelakte international gültige Normen unterworfen sind (das weitergehende völkerrechtliche Verbot der Apartheid läßt sich einstweilen nicht verallgemeinern), müßte ein solcher Versuch de lege lata scheitern.

Kühnhardt hat allerdings klare Vorstellungen über das angemessene Menschenrechtsverständnis: Um »angeborene « Rechte handele es sich, die "vorstaatlich" gälten; in ihrem Zentrum stehe der »individuell unverwechselbare Personalitätsbegriff“ (wohl: die Vorstellung, jeder Mensch sei kraft seiner Persönlichkeit unverwechselbar); mit der "Freiheitsbewegung" seien die Menschenrechte nicht identisch, würden jedoch am besten durch »parlamentarische Demokratie und Gewaltenteilung" verwirklicht. Zu Recht weist Kühnhardt diese seine Vorstellung nicht als völkerrechtlich vorgegeben aus, trägt sie aber mit dezidiertem Richtigkeitsanspruch vor. Das kann dann nur ein sozialwissenschaftlicher Richtigkeitsanspruch sein. Und in der Tat: Trotz rechtswissenschaftlicher Ausblicke und Exkurse versteht sich die Arbeit explizit vornehmlich als "zeit- und ideengeschichtliche Untersuchung". Als solche versucht sie den Nachweis zu erbringen, daß der aus Rinnsalen der griechisch-römischen Antike gespeiste, durch das Christentum in neue Bäche gegossene, in der europäischen Aufklärung angeschwollene Gedankenstrom, der sich spiegelt etwa in Normativakten des englischen 17., des amerikanischen und des französischen 18. Jahrhunderts, des deutschen 19. Jahrhunderts, einen »edlen Gedanken" hervorgebracht habe, der der heutigen Welt das Heil geben könne.

Jeder, dessen wissenschaftlicher Eros von europäischer Verfassungstradition und rechtsstaatlichem Gedankengut geprägt ist, wird Kühnhardt Glückauf auf dem Wege wünschen. Doch leider gelangt der Autor letztlich nicht ans Ziel. Das liegt zum einen, aber nicht entscheidend, daran, daß die Behauptung "vorstaatlicher" Geltung, der Umgang mit durch Geburt erworbenen, nicht durch staatlichen Willensakt verliehenen Rechten, die Perspektive verdunkelt. Das liegt ferner an der Abstraktheit »des « Menschenrechtsbegriffs: Der Nachweis, daß Recht Personen achten, ihre Würde respektieren, ihrem Freiheitsdrang Raum geben müsse, greift nicht weit genug. Niemand bestreitet es, nur das Wie steht im Streit. Und würden wir schließlich unterstellen, Kühnhardt sagte klarer, was der Inhalt des 
universell geltenden Normenensembles "die Menschenrechte" sein soll: Es bliebe zu klären, wie sie unter den so unterschiedlichen Bedingungen in den einzelnen Segmenten der Weltgesellschaft jeweils gleichermaßen segensreich wirken sollen, wie sie sich hineinfügen in unterschiedliche Traditionen und Entwicklungssituationen. Es genügen nicht die wenigen Worte zu den "Zukunftsperspektiven für die Menschenrechte», von denen der Autor meint, sie entzögen sich der wissenschaftlichen Analyse (S. 382). Wie Menschenrechten weltweit Beachtung verschafft werden kann, mag eine Frage sein, die auch spekulative Antworten erfordert (ohne deswegen von wissenschaftlicher Betrachtung ausgeschlossen zu sein!); $o b$ und welche Elemente alles dessen, was Kühnhardt "den Menschenrechten" zuordnet, unter welchen Bedingungen welche Konsequenzen zeitigen, muß beantwortet werden, ehe "Universalität» bejaht werden kann. Die Beweisführung zu liefern, die hier zu einer positiven Antwort führt, wäre eine Tat.

Kühnhardt sucht jedoch nicht nach Antworten auf solche Fragen, er unterstellt sie offenbar als ersichtlich. Das bleibt sympathischer Gestus, ist aber nicht mehr. Der Autor geht anders vor: Die Überlegenheit dessen, was als "für alle Menschen, zu allen Zeiten und unter allen historischen Bedingungen Gültigkeit" beanspruchend (S. 35) ausgewiesen wird, sucht Kühnhardt durch einen Vergleich hervortreten zu lassen. Das Denken aller Kulturen und Zeiten wird an "der Menschenrechtsidee" gemessen. Auch das begegnet methodischen Bedenken: Es wird nicht gef ragt, wie «außerwestliches « Denken um die Begrenzung staatlicher Herrschaft und den Schutz der Person gerungen hat; dem Autor reicht, daß er kein Pendant zum Menschenrechtsgedanken westlicher Prägung findet, um daran sein Postulat zu knüpfen: Mögen »die Menschenrechte» in die Lücken fließen, die das nicht-europäische Denken läßt.

Grundsätzliches also ist gegen die Anlage des Buches zu erinnern. Von seiner Durchführung soll hier nur kurz die Rede sein - sie ist bedingt durch die Weite der Begriffe und die Enge der Perspektive bei der Durchmusterung "außerwestlichen« Denkens. Bei seiner wahrhaft universellen Wanderung durch die Ideengeschichte streift der Autor nahezu alles, was dem ja nicht trennscharfen Begriff »Menschenrechte« zugeordnet werden kann. Aber er kann es nur streifen und mit einem aufwendigen Fußnotenapparat versehen. Er spricht wie erwähnt - über Völkerrecht und nationales Recht, auch über Friedenserhaltung und Legitimität der Herrschaft, über das Selbstbestimmungsrecht der Völker. Zentrale Kapitel diskutieren marxistisches Denken, »kosmisches Denken und Kastenordnung in Indien ", die "japanische Menschenrechtsstrategie», die geistesgeschichtliche Entwicklung Chinas, das Spannungsfeld von Freiheit und Entwicklung im Prozeß des afrikanischen Nation-buildings. Abschnitte über "Anfechtungen" der Menschenrechtsidee greifen u.a. neuere Schlagworte wie "Menschenrecht auf Frieden" u.ä. auf. Seitenhiebe auf den Zustand der "politischen Kultur" und den »Wertewandel« der Industriegesellschaft fehlen nicht. Dabei ist das ganze Buch durchaus flüssig geschrieben und verwertet eine Unmenge Sekundärliteratur, in den eigentlich interessanten Kapiteln über das außerwestliche Denken freilich vornehmlich solche. Wirkliche Schneisen in das vorgeführte gedankliche Dickicht werden nicht geschlagen, Belesenheit und Additionsfähigkeit scheinen unerschöpflich, manches wirkt hastig zusammengestellt. Daß »Die Europäische Menschenrechtskonvention . . zum 
bislang intensivsten Menschenrechtsschutz für das Individuum gefunden« hat, - unbestreitbar ist das richtig - belegt Kühnhardt mit einem eigenen Artikel in "Christ und Welt" von 1978.

Das Buch kann dem empfohlen werden, der Einzelinformationen und weiterführende Belege sucht; kein Sachverzeichnis, aber eine übersichtliche und detaillierte Gliederung erschließen die Fleißarbeit Kühnhardts für diesen Zweck. Für den Nachweis der „Universalität der Menschenrechte« hätte weniger bedeutend mehr sein können.

Philip Kunig

\section{amnesty international (Hrsg.)}

Der regionale Menschenrechtsschutz in Afrika, Amerika und Europa

Frankfurt/ Main: Fischer Taschenbuch Verlag, 1988, 291 Seiten, DM 16,80

Die Menschenrechte, so wird zu recht beklagt, sind in der politischen Auseinandersetzung vielfach zu billiger Münze geworden. Außerhalb wissenschaftlicher Publikationen wird nur selten unterschieden zwischen einzelnen Rechten, die der eine oder andere Staat verletzt haben soll. Teilweise wird es nicht einmal mit der Prüfung der Norm sehr ernst genommen oder getrost unterschlagen, daß die betreffende Norm entweder überhaupt keine Geltung besitzt oder nicht für den betreffenden Staat. Damit steht jedoch auch die Glaubwürdigkeit der Kritik in Frage.

$\mathrm{Daß}$ das, was für die politische Öffentlichkeit zur Normalität geworden ist, nicht tatenlos hingenommen werden muß, auch wenn es nur in kleinen Schritten geändert werden kann, beweist das vorliegende Buch, mit dem sich amnesty international (ai) an eine breite Öffentlichkeit wendet. Aus verschiedenen Gründen ist es geeignet, eine intensivere Beschäftigung mit dem internationalen Menschenrechtsschutz zu fördern.

Die handliche Taschenbuchausgabe versteht sich als Ergänzung zu dem im Jahre 1981 in demselben Verlag erschienen Buch Der internationale Menschenrechtsschutz, das sich mit Deklarationen und Konventionen der Vereinten Nationen beschäftigt. Nunmehr wendet sich ai dem regionalen Menschenrechtsschutz zu, der bisher in den drei im Titel genannten Kontinenten komplexe normative und institutionelle Ausformungen gefunden hat.

Die Ausgabe lebt aus ihrem umfangreichen Anhang heraus, der neben den drei Menschenrechtskonventionen [ein Erratum: Die Afrikanische Charta datiert von 1981.] eine Reihe bedeutender Dokumente, jeweils in zumeist nicht-amtlicher Übersetzung und teils im Auszug, leichter zugänglich macht. Dies gilt insbesondere für die Afrikanische Flüchtlingskonvention vom 10. 9. 1969, die Amerikanische Menschenrechtserklärung vom 2. 5. 1948, die KSZE-Schlußakte vom 1. 8. 1975 und das Dritte AKP-EWG-Abkommen vom 8. 12. 1984 (Lomé III). Die Übersetzungen - auch die amtlichen - sind nicht authentisch, dienen aber ohne Frage der besseren Zugänglichkeit der Dokumente. Wichtig dafür auch das Verzeichnis der Vertragsstaaten im Anschluß an die jeweiligen Verträge. 